\title{
New Direction for Enhancing Quality in Diabetes Care: Utilizing Telecommunications and Paraprofessional Outreach Workers Backed by an Expert Medical Team
}

Penelope Ann Mayes, R.N., B.S.N., P.H.N., C.D.E., ${ }^{1}$ Abraham Silvers Ph.D., ${ }^{2}$ and J. Joseph Prendergast, M.D. ${ }^{1}$

${ }^{1}$ Endocrine Metabolic Medical Center, Palo Alto, California. ${ }^{2}$ University of California Medical School at San Francisco, San Francisco, California.

\section{Abstract}

This article assesses the value of using telecommunications with Promatoras (paraprofessional outreach workers) and an expert medical team of registered nurses (RNs) and endocrinologists in an at-risk type 2 diabetic Hispanic population recruited for a telemedicine feasibility project from a free clinic. Nineteen patients agreed to enter the program and 16 completed the program in 3.5 years of study. A Promatoras is the primary educator and the point of communication to patient or medical personnel overseeing each patient's home glucose monitoring, medical records, and medications, regularly communicating by telephone and e-mail with patients and diabetes specialists. Between clinic visits, all routine care, including body weight, blood glucose, and blood pressure monitoring, was shared over the Internet, and each patient was interviewed by audio and camera. The endocrinologist was in his office, while the primary care physician, patient, and Promotora volunteers were at the free clinic. Four variables were considered in this longitudinal study: weight, systolic blood pressure, diastolic blood pressure, and HbA1c. Estimates of means, correlations, t-tests, and slopes of the repeated measures were obtained, and comparisons were made between first and last values. The most important sign of improvement in the patients' situation was the significant decrease in $\mathrm{HbAlc}$ to $7.2 \%$ from $9.6 \%(\mathrm{p}=0.001)$.

Key words: disease management, diabetes, telemmmedicine, home health

\section{Introduction}

$\mathrm{T}$ he purpose of this article was to demonstrate the value of using telecommunications with Promatoras (paraprofessional outreach workers) and an expert medical team of registered nurses (RNs) and endocrinologists in a free clinic in San Jose, CA, in the management of diabetes in an at-risk Hispanic population.

There is an evolving crisis that limits the ability of the nation's healthcare delivery system to provide adequate care for type 2 diabetic patients. This crisis is caused by three factors: the growing diabetes epidemic, particularly in young people ${ }^{1}$; the shortage of practicing endocrinologists ${ }^{2}$; and the impending shortage of primary care physicians. $^{3,4}$

The rapid rise in the incidence of type 2 diabetes has been noted in the literature. Estimates of its prevalence in the United States were 23.6 million in 2007, an increase of more than 3 million from 2005 estimates. The total prevalence of diabetes increased by $5 \%$ from 2005 to 2007 and by $24 \%$ since 2002 . $^{5}$ 


\section{ENHANCING DIABETES CARE UTILIZING TELECOMMUNICATIONS}

With prospective changes in insurance legislation, there will be more diabetic patients covered by insurance and expecting medical care.

Richard Hellman, President of the American Association of Clinical Endocrinologists (AACE), noted in the Philadelphia Inquirer that AACE predicts a 25-35\% shortage of endocrinologists by $2020 .^{2}$ Practicing endocrinologists will retire, and there will be fewer new physicians entering the field. An appointment with an endocrinologist could require a long wait. If several million additional patients, presently uninsured or underinsured, are covered by some form of universal health insurance as appears likely, this will strain the medical system even more.

The American College of Endocrinology has consistently acknowledged the shortage of endocrinologists, particularly those trained in pediatric diabetes care. ${ }^{6}$ The American Diabetes Association (ADA) has noted that the number of physicians trained in clinical diabetes care has not kept pace with the nation's needs. The American Academy of Family Physicians projects a shortage of primary care providers who have traditionally cared for most people with diabetes. ${ }^{7}$

Estimates are that the United States will need 39\% more primary care physicians in the next 14 years and patients will be required to travel further to get medical help. Because primary care physicians are no longer able to care for all patients, people with diabetes and others with both acute and chronic illnesses are forced to seek care in hospital emergency rooms (ERs). After expanding health coverage to all residents of the state, Massachusetts has faced this very problem. ${ }^{8}$

The lack of trained diabetes experts and providers is only one aspect of the problem-a more fundamental issue is the currently limited structure for delivering complete and adequate healthcare.

New healthcare delivery options are needed to address these acute and interrelated problems, especially in those with diabetes. Telecommunication will likely provide one of these options ${ }^{9}$ and should be a major goal, especially given the extremely large number of diabetic patients. Travel time, costs, and inconvenience are minimized when healthcare comes to the patient. Communicating on a video screen over long distances diminishes inhibitions. For example, a recent study from the Mayo Clinic showed that video consultation improved secondary prevention of stroke, and that stroke telemedicine improved the treatment of acute stroke in rural areas. ${ }^{9}$

Underserved and underinsured patients are most affected; the Hispanic community is particularly affected. The prevalence of diabetes in Mexican Americans in the United States is reaching almost epidemic proportions. ${ }^{10}$ Approximately $18 \%$ of Mexican-American adults between the ages 50 and 64 have been diagnosed with diabetes-more than twice the rate for Caucasians (8.3\%). A recent
Pew Hispanic Center study showed that this population is poorly managed for two reasons: low rates of daily glucose monitoring and low medication use. ${ }^{10}$ When people with diabetes are inadequately treated and not managed on a regular basis, they develop preventable complications with greater frequency. For example, MexicanAmericans with diabetes are more prone than Caucasians to eye and kidney complications. Such preventable complications require costly care and cause unnecessary suffering. ${ }^{11}$

The problem for the Mexican-Americans in particular is twofold: limited access to primary medical care and severely restricted availability of the specialized diabetes care required to meet modern treatment standards. ${ }^{12}$ Primary care physicians find it difficult to stay abreast of the new technology and methods of treatment required to keep their knowledge and skills in diabetes care current. Even when Mexican-Americans with diabetes are able to obtain primary care, it is unlikely to meet new diabetes treatment standards. Access to quality, high-level care now demands that patients are educated about diabetes and assume a major role in their own case management. These requirements are often compromised in the MexicanAmerican community because of language and cultural barriers. ${ }^{13}$

On a positive note, the Hispanic population has a unique community support system. A group of volunteers of all ages and training, called Promatoras (health promoters), have served individuals in the community for generations, at all levels of need, including language translation, social customs, and financial transactions. This long-standing social network has functioned well in solving multiple social problems, as well as the problem of obtaining meaningful medical care.

Recently, increased training in diabetes care and general health has become available to Promatoras through a rigorous, formal curriculum available in San Francisco Bay Area community colleges and universities. With training and certification, a novel group of healthcare specialists in diabetes and general healthcare has emerged. These Promatoras provide medical support to medically illiterate, monolingual, and low economic status Hispanic patients. As these providers are from the same ethnic community, they easily establish rapport with patients and are able to translate medical instructions, provide social interpretation, and promote adherence to treatments.

With the Promatoras-Telemedicine Care Provider interaction model adopted from the Mayo Clinic Stroke Model, ${ }^{9}$ it is now possible for Hispanic diabetic patients to establish an effective relationship with providers skilled in diabetes care, a significant clinical and economic benefit. These Promatoras have assumed an active and central role as mentors and facilitators in day-to-day diabetes management and self- 
care of Hispanic patients. They utilized a telemedicine system, including person-to-person Internet video and telephonic communications, to maintain personal contact with patients.

\section{Materials and Methods}

The Hispanic patients recruited in this telemedicine feasibility project were type 2 patients of the RotaCare Health Clinic (RH Clinic) in Santa Clara County, CA. Nineteen patients agreed to enter the program and 16 completed the program. There were 3 men and 13 women. Ages ranged from $28(n=1)$ to $78(n=1)$ with the majority of patients in their 40s $(n=5)$, their 50s $(n=4)$, and their 60s $(n=3)$. The duration of the project was 3.5 years.

A Promatoras explained the organization of the care program to the patient. Because several Promatoras were based in the RH Clinic, a different person saw each patient during clinic visits. Between clinic visits, all routine care, including body weight, blood glucose, and blood pressure monitoring, was shared over the Internet, and each patient was interviewed by audio and camera. The endocrinologist was in his office, while the primary care physician, patient, and Promatora volunteers were at the RH Clinic. Promatoras provided the primary education and outreach staff, while the medical staff made and implemented care-related decisions.

Promatoras had also received additional training that qualified them to extend their role into the area of patient care. With this approach, the Promatoras assume an active and central role as mentors and facilitators in the day-to-day management of diabetes and self-care because they were bilingual, bicultural members of the Hispanic community. This allowed them to be effective facilitators and peer educators. They were also present at each patient telemedicine session, where they served as translators. They oversaw each patient's home glucose monitoring, medical records, and medications and regularly communicated, by telephone and e-mail, with the patient and with the diabetes specialists. They made regular home visits and instructed the patients and their families in the basic principles of a diabetic diet, weight control, and regular exercise. In addition, they communicated weekly, and met monthly, with the case manager to ensure that domestic issues that might compromise each participant's compliance were addressed and resolved. There were a lot of stressful issues, including relative's deaths, employment changes, benefit changes, and other domestic/social issues that could undermine continued compliance. Records of the sessions were sent via fax to the medical clinic to be incorporated into the patients' medical records.

An RN certified diabetes educator (CDE) advised the Promatoras and the patient about medical changes, laboratory and X-ray results, and the need, if necessary, for further consultation, such as surgery. Because the level of understanding of Promatoras and patient varied, 15-30 min were allowed per visit at the RH Clinic. If more time was needed, an additional appointment was scheduled for 1 week later.

In the 3.5 years of this program, only one patient needed consultation outside the clinic (for surgical gall bladder removal performed at a local hospital).

\section{STATISTICAL EVALUATION OF LONGITUDINAL DATA}

Data were analyzed using the S-PLUS ${ }^{\circledR}$ statistical software package, Professional Version 8 for Windows (Insightful Corporation, Seattle, WA). Patient data obtained during the first clinic visit served as a baseline and subsequent visits were from 1 week to 3 months apart, depending on the severity of their clinical situation. All 16 patients required several visits during this study, pointing to the value of close, regular communication between the patient and the medical team. Four variables were considered in this longitudinal study: weight, systolic blood pressure, diastolic blood pressure, and $\mathrm{HbA1c}$. Estimates of means, correlations, $t$-tests, and slopes of the repeated measures were obtained, and comparisons were made between first and last values.

\section{Results}

All patients were managed according to ADA standards. Retinal exams were done annually at the RH Clinic. Promatoras completed foot checks and general exams, checked blood pressure, and recorded all vital signs at each visit.

Telecommunications allowed frequent patient "visits." Each visit over the Internet, whether it was weekly or monthly, included inquiry about the role of diet and appropriate exercise by the expert nurse RN (Penelope Ann Mayes, R.N., B.S.N., P.H.N., C.D.E.), who provided leadership, education, and encouragement to the patient, to the Promatoras, and to all family and social support individuals playing a role in the patient's life. This amounted to 36-48 "classes on healthy living" in the 3.5 years of the program.

For appropriate medication adjustments, pharmaceutical companies graciously provided sample medications:

- NPH insulin obtained from multiple sources, low doses of Glyburide, and low doses of Avandamet were used.

- The reduction in insulin to an A.M. dose only was able to be achieved in several patients.

- Hypertension control was maintained with the drugs Lotrel, Norvasc, or Diovan, as needed.

- Lipid control was maintained with either Pravachol or Zocor (40 mg daily during the study). 


\section{ENHANCING DIABETES CARE UTILIZING TELECOMMUNICATIONS}

Table 1. Baseline Values in Patients

BODY

WEIGHT

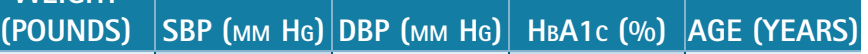

\begin{tabular}{l|l|l|l|l|}
\hline $166(6.9)$ & $134.6(4.5)$ & $81.8(2.7)$ & $9.6(0.6)$ & $51(2.5)$ \\
\hline
\end{tabular}

SBP, systolic blood pressure; DBP, diastolic blood pressure.

Note: Mean and standard errors at baseline.

Laboratory data, obtained by Promatoras, were inserted in the chart and sent to the RN CDE, along with a record of questions and patient replies about the patient's health since last seen, other physicians seen by the patient, and if the patient required a visit to an ER or hospital. Positive answers were followed up on by the RN CDE. Except for occasional upper-respiratory symptoms and one cholestectomy, there were no other ER or hospital visits. The patient measurements at initial visit are summarized in Table 1.

There were no clinical signs or symptoms of complications except for the elevated HbA1c. During the 3.5 years of this study there was no deterioration in blood pressure control and only a modest ( 5 pound) increase in body weight ( $p=0.03$ ). The most important sign of improvement in the patients' situation was the significant decrease in HbA1c (Table 2). The difference between the last and first value for HbA1c (mean 7.2\% vs. 9.6\%, respectively) was $-2.4 \%$ ( $p=0.001$; a decrease of 21\%) and slope of the repeated measures was $r=0.65$ $(p<0.05)$. Finally, Table 3 shows that very little or no change occurred in weight or systolic and diastolic blood pressure.

\section{Discussion}

Four conclusions can be drawn from these data:

- Video conferencing via the Internet can provide a useful tool to assure that patients who adopt and utilize ADA protocols for diabetes will improve their glucose control.

- The protocols can be executed by Promatoras with the support and oversight of a RN CDE and an endocrinologist, when needed. Only one question was asked of the endocrinologist during the total of 3.5 years.

- The protocol was successfully executed by the Promotora as evidenced by changing or decreasing medications as needed. Patients were as fully educated as possible by the RN CDE.

- Finally, patients (and their family members) responded positively to the need for understanding the medical challenges of diabetes, by participating in their own care, and by returning, without complaint, for additional visits, when the need was

\begin{tabular}{|c|c|c|c|c|c|}
\hline PATIENT & $\begin{array}{c}\text { BASELINE } \\
\text { HвA1c }\end{array}$ & $\begin{array}{l}\text { FINAL } \\
\text { HBA1c }\end{array}$ & $\begin{array}{c}\text { DIFFER- } \\
\text { ENCE }\end{array}$ & $\begin{array}{c}\text { PERCENT } \\
\text { DIFFER- } \\
\text { ENCE }\end{array}$ & SLOPE \\
\hline 2 & 10.4 & 5.7 & -4.7 & -45.19 & -0.1 \\
\hline 3 & 6.7 & 6.7 & 0 & 0 & 0.02 \\
\hline 4 & 6.5 & 5.9 & -0.6 & -9.23 & -0.04 \\
\hline 5 & 10.5 & 8 & -2.5 & -23.81 & -0.02 \\
\hline 6 & 11.3 & 6.6 & -4.7 & -41.59 & -0.08 \\
\hline 7 & 8.2 & 7 & -1.2 & -14.63 & -0.04 \\
\hline 8 & 8.4 & 7.6 & -0.8 & -9.5 & 0 \\
\hline 9 & 12.1 & 6.2 & -5.9 & -48.76 & -0.82 \\
\hline 10 & 14.2 & 11.3 & -2.9 & -20.42 & -0.06 \\
\hline 12 & 14.4 & 7.7 & -6.7 & -46.53 & -0.58 \\
\hline 13 & 7.3 & 7 & -0.3 & -4.11 & 0 \\
\hline 14 & 6.4 & 7.7 & 1.3 & 20.31 & 0.04 \\
\hline 15 & 10.3 & 7 & -3.3 & -32.04 & -0.22 \\
\hline 16 & 11.1 & 7.1 & -4 & -36.04 & -0.07 \\
\hline 17 & 10.6 & 7.5 & -3.1 & -29.25 & -0.07 \\
\hline 18 & 5.6 & 5.9 & 0.3 & 5.36 & 0.01 \\
\hline
\end{tabular}

Note: $\mathrm{HbA} 1 \mathrm{C}$ for individual patients at baseline and after 3.5 years. Slope of repeated measures.

identified. The model shown in Figure 1 proved to be effective in providing improved healthcare delivery to a Hispanic population subgroup.

Caucasians will become a minority in the United States in approximately 30 years, according to the U.S. Census Bureau and by then the majority of the U.S. population will be composed of

\section{Table 3. Effect of Program After 3.5 Years}

\begin{tabular}{|l|l|l} 
WEIGHT (LBS) & SBP (MM HG) & DBP (MM HG)
\end{tabular}

\begin{tabular}{l|l|l}
$+5.0, p=0.03$ & $+5.6, p=0.28$ & $+0.5, p=0.9$
\end{tabular}

Note: Mean change of body weight, SBP, and DBP from baseline to final measurements at 3.5 years. 


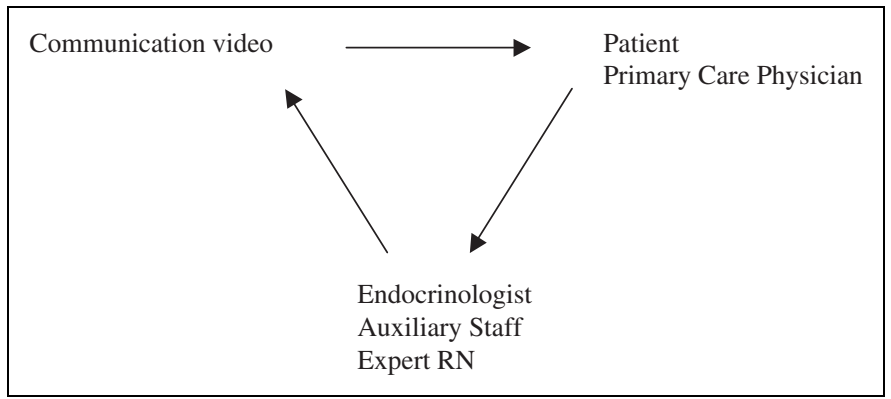

Fig. 1. A model of interaction between patients and healthcare providers.

Hispanic, African American, Asian, Native American, Native Hawaiian, and Pacific Islanders. These population groups currently comprise a huge proportion of the uninsured and are highly susceptible to diabetes. The model provided here was used to interact with uninsured Hispanics and may well be a meaningful approach for maintaining the health of other minority groups.

This study used a decades-old, trusted form of education and social communication in healthcare, an approach used previously. ${ }^{13}$ An outcome using a similar approach in Hong Kong has recently been published. ${ }^{14}$ With new technology platforms such as YouTube, Face Book, and Twitter, the ability to provide rapid responses to changes in patients' health may become commonplace. When larger numbers of patients with a similar disease such as diabetes participate in their own care they develop a collective insight that, when shared with others, can exceed the wisdom of any single patient or physician.

This approach does not exclude expert opinion; it utilizes opinion from multiple expert sources. ${ }^{13}$ In addition, healthcare delivery systems need not be disruptive. New pathways for quality care, involving multiple innovative systems in healthcare, especially at lesser cost, have been suggested. ${ }^{15,16}$ These systems expect patients to take an interest in their own case and be persistent and active in their own healthcare.

The results of the present program demonstrate the results of that interest and activity at a personal level. The value of lowering HbA1c to prevent diabetes-related complications was advocated some 10 years ago. ${ }^{17}$ Patients who participated in our program were able to lower HbA1c by over 20\% and suffered no disease-related complication.

The Institute for Health Care Improvements ${ }^{16}$ underscored the need for patients taking part in shared decisions for their medical care. It was pointed out the usefulness of the Internet to help them access quality information. The success of the patient management style presented here is independent of the medications used and relies heavily on a social framework that has been part of the Hispanic community since early in the last century. It is notoriously difficult to keep in touch with the poor, particularly if they are illegal immigrants. The New Technologies of Social Media should find a ready acceptance in similar populations. Patients will be able to collaborate with other patients, caregivers, and stakeholders in their health. They should be more interested in sharing the collective wisdom of their communities by establishing healthcare networks enabling all to find information "not known to be out there."

Patient opinion leaders are now established for other chronic disease states, such as HIV, mental health, and cancer. This approach does not deny the value of expert opinion, but the source of expertise need not be limited to only one opinion. As other novel approaches for providing improved outcomes are enlisted, the challenges and opportunities of a new social context must be considered. ${ }^{16}$

\section{Acknowledgments}

The authors thank the staff and patients at RH Clinic who cooperated in this lengthy study and the pharmaceutical companies that provided the drugs used.

\section{Disclosure Statement}

No competing financial interests exist.

\section{REFERENCES}

1. Hitti M. Nearly 24 million have diabetes, CDC reports a steep rise in diabetes patients in U.S. [reviewed by Louise Chang, MD; article online]. WebMD Health News, 2008. Available at http://diabetes.webmd.com/news/20080624/ nearly-24-million-have-diabetes (last accessed July 31, 2009).

2. Levy P. Endocrinologist shortage a growing problem [article online]. FierceHealthcare 2006. Available at www.fiercehealthcare.com/story/ endocrinologist-shortage-a-growing-problem/2006-09-13 (last accessed July 31, 2009).

3. O'Reilly KB, Hedger B. Doctors urge: Rescue primary care or work force shortage will mount [article online]. Am Med News 2008. Available at www.amaassn. org/amednews/2008/12/08/prl11208.htm (last accessed July 31, 2009).

4. Duncan K. Addressing primary care physician shortage is key to health care reform [article online]. San Mateo County Med Assoc 2009;LXIV. Available at www.smcma.org/bulletin/issues/Feb09.pdf (last accessed July 31, 2009).

5. American Diabetes Association. Diabetes statistics, 2009. Available at www.diabetes.org/diabetes-statistics.jsp (last accessed July 31, 2009).

6. Dixon BK. With specialist shortage, obese kids turn to PCPs: The data suggest that geographic differences in endocrinologist supply may be driving variation [article online]. Fam Pract News 2008;38:42. Available at www. 


\section{ENHANCING DIABETES CARE UTILIZING TELECOMMUNICATIONS}

familypracticenews.com/article/S0300-7073(08)70602-9/preview (last accessed July 31, 2009).

7. Physicians group predicts family doctor shortage in at least five states by 2020 [article online]. Med News Today, 2006. Available at www.medicalnewstoday .com/articles/52884.php (last accessed July 31, 2009).

8. Sack K. In Massachusetts, Universal Coverage Strains Care [article online]. The New York Times, 2008. Available at www.nytimes.com/2008/04/05/ us/05doctors.html (last accessed July 31, 2009)

9. Demaerschalk BM, Miley ML, Kiernan TE, Bobrow BJ, Corday DA, Wellik KE, Aguilar MI, Ingall TJ, Dodick DW, Brazdys K, Koch TC, Ward MP, Richemont PC STARR Coinvestigators. Stroke telemedicine. Mayo Clin Proc 2009;84:53-64. Available at www.mayoclinicproceedings.com/content/84/1/53.abstract (last accessed July 31, 2009).

10. Rosenbloom AL, Joe JR, Young RS, Winter WE. Emerging epidemic of type 2 diabetes in youth. Diabetes Care 1999;22:345-354.

11. Harris MI, Klein R, Cowie CC, Rowland M, Byrd-Holt DD. Is the risk of diabetic retinopathy greater in non-Hispanic blacks and Mexican Americans than in non-Hispanic whites with type 2 diabetes? A U.S. population study. Diabetes Care 1998;21:1230-1235.

12. Pugh JA, Stern MP, Haffner SM, Eifler CW, Zapata M. Excess incidence of treatment of end-stage renal disease in Mexican Americans. Am J Epidemiol 1998;127:135-144.

13. Sarasohn-Kahn J. The wisdom of patients: Health care meets online social media. California HealthCare Foundation; Chronic Disease Care, 2008. Available at www.chcf.org/topics/chronicdisease/index.cfm?itemID $=133631$ (last accessed July 31, 2009).

14. Chan JC, So WY, Yeung CY, Ko GT, Lau IT, Tsang MW, Lau KP, Sin SC, Li JK, Yeung VT, Leung WY, Tong PC; SURE Study Group. Effects of structured versus usua care on renal endpoint in type 2 diabetes: the SURE study: a randomized multicenter translational study. Diabetes Care 2009;32:977-982.

15. Varkey $P$, Horne $A$, Kevin $E$, Bennet KE. Innovation in health care: A primer. Am J Med Qual 2008;23:382-388.

16. IHI.org. A resource from the Institute of Healthcare Improvement. Percentage of patients with documented self-management goals. Available at www.ihi.org/ IHI/Topics/ChronicConditions/Diabetes/Measures/DocumentedSelfmanagementGoals.htm (last accessed July 31, 2009).

17. de Lissovoy G, Ganoczy DA, Ray NF. Relationship of hemoglobin A1c, age of diabetes diagnosis, and ethnicity to clinical outcomes and medical costs in a computer-simulated cohort of persons with type 2 diabetes. Am J Manag Care 2000;6:573-584.

Address correspondence to:

J. Joseph Prendergast, M.D.

Endocrine Metabolic Medical Center 350 Cambridge Ave., Suite 250 Palo Alto, CA 94306

E-mail: office@endocrinemetabolic.com

Received: August 5, 2009

Revised: October 13, 2009 Accepted: October 13, 2009 